\title{
Optimizing cutting parameters in hard turning of AISI 52100 steel using topsis approach
}

Ponugoti UMAMAHESWARRAO, D. RANGA RAJU, Koka Naga Sai SUMAN, B. RAVI SANKAR

DOI: 10.30464/jmee.2019.3.3.227

Cite this article as:

Umamaheswarrao P. et al. Optimizing cutting parameters in hard turning of AISI 52100 steel using topsis approach. Journal of Mechanical and Energy Engineering, Vol. 3(43), No. 3, 2019, pp. 227-232.

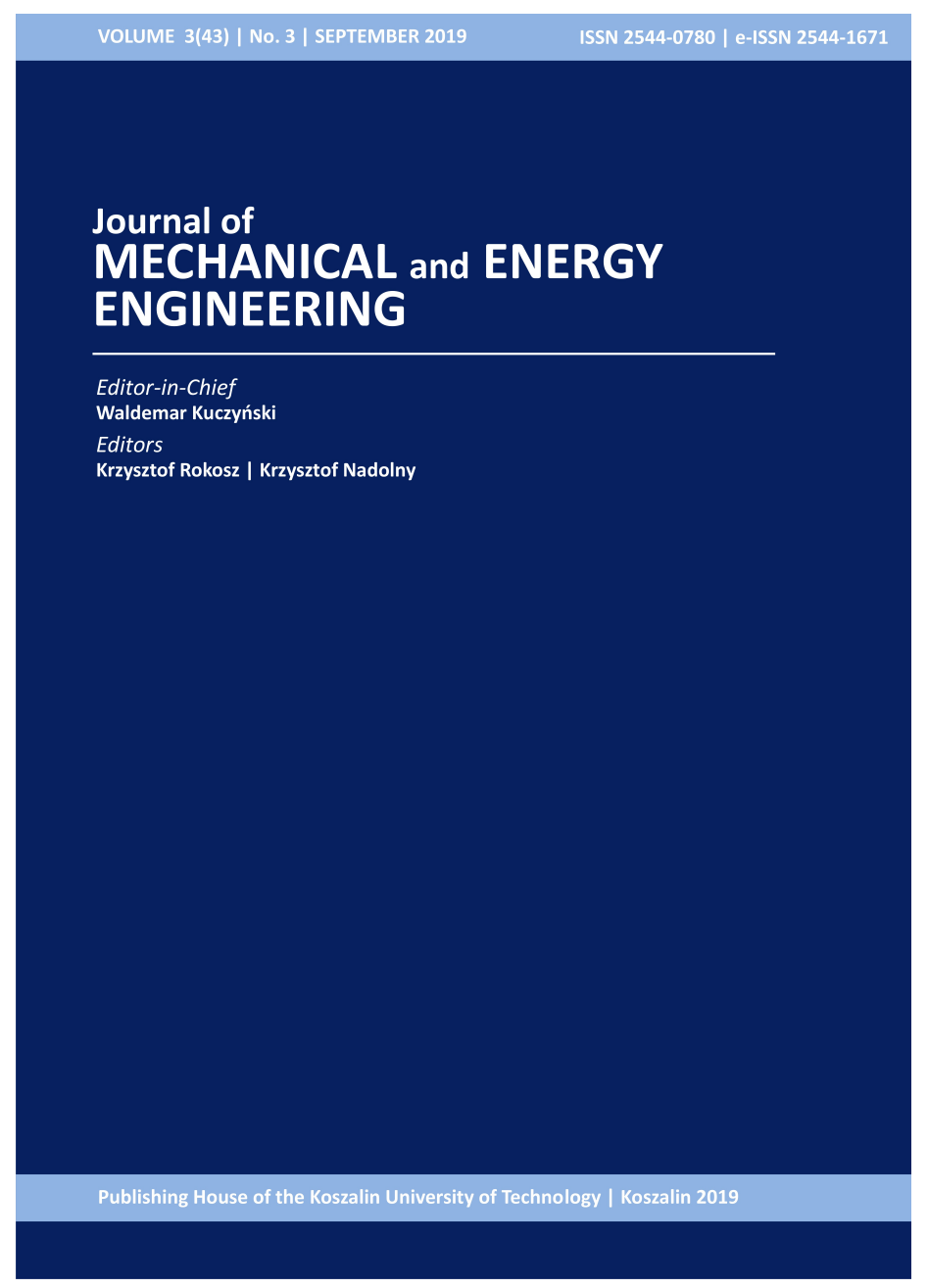
Journal of Mechanical and Energy
Engineering

Website: jmee.tu.koszalin.pl

ISSN (Print): 2544-0780

ISSN (Online): 2544-1671

Volume: 3(43)

Number: 3

Year: 2019

Pages: 227-232

Article Info:

Received 21 July 2019

Accepted 21 October 2019

\section{Open Access}

This article is distributed under the terms of the Creative Commons Attribution 4.0 (CC BY 4.0) International License (http://creativecommons.org/licenses/by/4.0/), which permits unrestricted use, distribution, and reproduction in any medium, provided you give appropriate credit to the original author(s) and the source, provide a link to the Creative Commons license, and indicate if changes were made. 


\title{
OPTIMIZING CUTTING PARAMETERS IN HARD TURNING OF AISI 52100 STEEL USING TOPSIS APPROACH
}

\author{
Ponugoti UMAMAHESWARRAO ${ }^{1 *}, 2$, D. RANGA RAJU ${ }^{3}$, Koka Naga Sai SUMAN ${ }^{4}$, \\ B. RAVI SANKAR ${ }^{5}$ \\ 1* Research Scholar, Department of Mechanical Engineering, Andhra University College of Engineering, \\ Visakhapatnam, A.P. India, e-mail: maheshponugoti@gmail.com \\ ${ }^{2}$ Department of Mechanical Engineering, Bapatla Engineering College, Bapatla, A.P. India \\ ${ }^{3}$ Department of Mechanical Engineering, Srinivasa Institute of Engineering and Technology, Amalapuram, \\ A.P. India \\ ${ }^{4}$ Department of Mechanical Engineering, Andhra University College of Engineering, Visakhapatnam, A.P. India \\ ${ }^{5}$ Department of Mechanical Engineering, Bapatla Engineering College, Bapatla, A.P. India
}

(Received 21 July 2019, Accepted 21 October 2019)

\begin{abstract}
In the present work optimization of cutting parameters is performed while hard turning of AISI 52100 steel with polycrystalline cubic boron nitride (PCBN) tools using Technique for Order Preference by Similarity to Ideal Solution (TOPSIS). Experiments are planned and conducted based on Center Composite Rotatable Design (CCD) of the Response Surface Method (RSM). Cutting speed, feed, depth of cut, nose radius and negative rake angle are considered as input parameters. In this study machining force (F) and surface roughness (Ra) are measured during the experiment. Analysis of variance (ANOVA) is deployed to determine the influence of process parameters. Obtained optimal parameters are speed $200 \mathrm{rpm}$, feed $0.1 \mathrm{~mm} / \mathrm{rev}$, depth of cut $0.8 \mathrm{~mm}$, nose radius $1.2 \mathrm{~mm}$ and negative rake angle $45^{\circ}$.
\end{abstract}

Keywords: machining force, surface roughness, TOPSIS, optimization

\section{INTRODUCTION}

Hard turning evolved as an improved machining process incontrast to grinding due to numerous merits such as process flexibility, economic, less setup time, complex parts fabrication and absence of coolant [1-2]. AISI 52100 steel was widely accepted material for abundant applications such as bearings, rollers, and dies etc and the turning process was inevitable for the aforementioned applications. Optimal process parameters selection was essential for higher-order machining performance, Multi criteria decision making methods (MCDM) were proved as tools in several manufacturing applications [3]. Among many TOPSIS method was adopted and gained acceptance for optimizing machining parameters [4].

Himadri Majumder and Abhijit Saha [5] optimized process parameters in turning of ASTM A588 mild steel using a hybrid optimization tool i.e. MOORAPCA and TOPSIS-PCA approach. Tian [6] used
TOPSIS (Technique for Order Preference by Similarity to Ideal Solution) for optimization of input parameters in CNC machining of S45C steel. Palanisamy and Senthil [7] carried out of process parameters optimization in turning of 15-5 $\mathrm{PH}$ stainless steel using Taguchi based Grey approach and TOPSIS. It is concluded that force and surface roughness are predominantly affected by feed rate.

Maheswararao and Venkata subbaiah [8] employed TOPSIS for optimization of process parameters in the CNC machining of AA7075. Results concluded that feed rate has a significant influence on responses. Sagar Bhise et al. [9] studied the effect of input parameters on surface roughness in hard turning of M42 austenitic stainless steel using CBN and carbide inserts by deploying PCR-TOPSIS. Maity and Khan [10] determined an optimal combination of process parameters during turning of commercially pure titanium (CP-Ti) grade 2 using the MCDM-based TOPSIS method. 
Singaravel et al. [11] optimized machining parameters and nose radius in turning of EN25 steel by the application of combined MOORA and entropy measurement method. Singaravel et al. [12] determined optimum process parameters using the Additive Ratio Assessment (ARAS) method in turning of AISI 4340 steel. Optimization of process parameters is performed using various techniques like GRA-PCA [13-15], GA [16], ANN [17], TOPSIS [18-19]. Hence, the present work aimed to optimize process parameters for AISI 52100 steel hard turning using TOPSIS.

\section{EXPERIMENTAL DETAILS}

Machining details and experimental matrix with responses are shown in Table 1 and Table 2 respectively. The experimental setup is depicted in Figure 1. In the current study, Kirloskar Turn Master35 type lathe was employed for conducting experiments in dry condition and AISI 52100 steel was deployed as a workpiece having a diameter of 48 $\mathrm{mm}$ and length of $500 \mathrm{~mm}$. For this experimentation, five process variables are chosen such as Cutting Speed, Feed, Depth of cut, Nose radius, and Negative rake angles. PCBN tools with designation (CNMG 120404, CNMG 120406, CNMG 120408, CNMG 120410, CNMG 120412) manufactured by Zen Diamond Tools, Chennai, India are depicted in Figure 2.

Tab. 1 Machining Conditions

\begin{tabular}{|c|c|c|}
\hline Designation & Notation & $\begin{array}{l}\text { Adopted for the present } \\
\text { study }\end{array}$ \\
\hline $\begin{array}{l}\text { Workpiece } \\
\text { material }\end{array}$ & & AISI 52100 steel \\
\hline Dimensions & & $\begin{array}{l}48 \mathrm{~mm} \text { diameter and } 500 \mathrm{~mm} \\
\text { length }\end{array}$ \\
\hline Hardness & & $57 \mathrm{HRC}$ \\
\hline $\begin{array}{l}\text { Cutting speed } \\
(\mathrm{rpm})\end{array}$ & $v$ & $200,400,600,800,1000 \mathrm{rpm}$ \\
\hline $\begin{array}{l}\text { Feed } \\
(\mathrm{mm} / \mathrm{rev})\end{array}$ & $f$ & $\begin{array}{l}0.02,0.04,0.06,0.08,0.1 \\
\mathrm{~mm} / \mathrm{rev}\end{array}$ \\
\hline $\begin{array}{l}\text { Depth of cut } \\
(\mathrm{mm})\end{array}$ & $d$ & $0.4,0.5,0.6,0.7,0.8 \mathrm{~mm}$ \\
\hline Nose radius & $r$ & $0.4,0.6,0.8,1,1.2 \mathrm{~mm}$ \\
\hline $\begin{array}{l}\text { Negative rake } \\
\text { angle }\end{array}$ & $\alpha$ & $5,15,25,35,45$ \\
\hline $\begin{array}{l}\text { Cutting } \\
\text { environment }\end{array}$ & & Dry \\
\hline $\begin{array}{l}\text { Cutting } \\
\text { inserts }\end{array}$ & & $\begin{array}{l}\text { Polycrystalline cubic } \\
\text { boron nitride (PCBN) }\end{array}$ \\
\hline Tool holder & & PSBNR 2525 M12 \\
\hline $\begin{array}{l}\text { Tool } \\
\text { geometry }\end{array}$ & & $\begin{array}{l}\text { CNMG120404, CNMG120406, } \\
\text { CNMG120410, CNMG120412 }\end{array}$ \\
\hline $\begin{array}{l}\text { Machining } \\
\text { length }\end{array}$ & & $30 \mathrm{~mm}$ \\
\hline Responses & $\begin{array}{l}F_{m} \\
R a\end{array}$ & $\begin{array}{l}\text { Machining force, } \\
\text { Arithmetic mean roughness }\end{array}$ \\
\hline
\end{tabular}

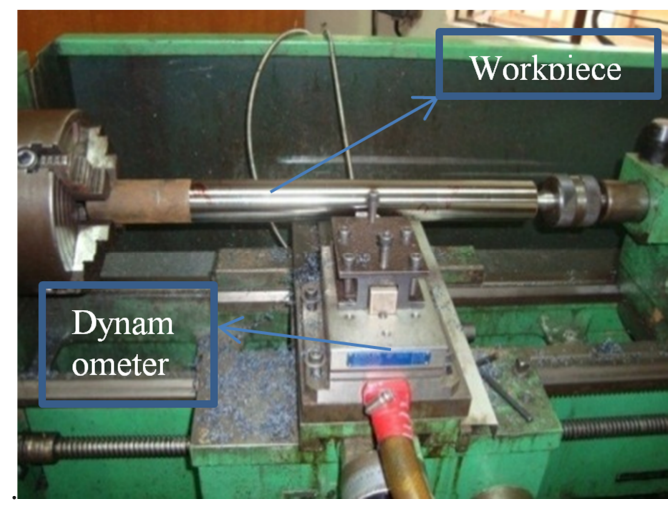

Fig. 1. Experimental setup

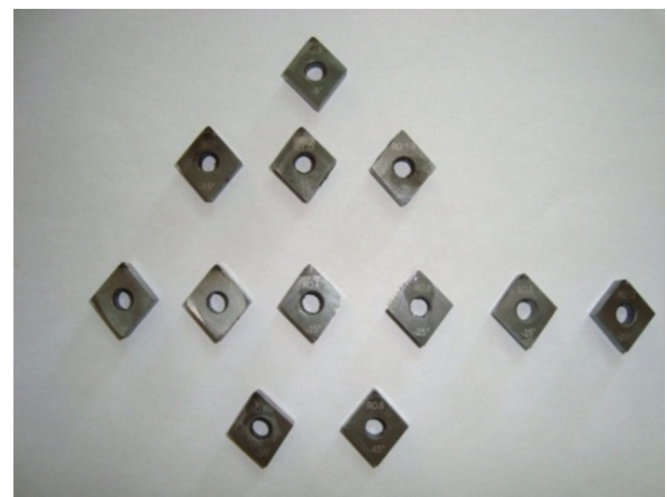

Fig. 2. PCBN tools

\section{TECHNIQUE FOR ORDER OF PREFERENCE BY SIMILARITY TO IDEAL SOLUTION (TOPSIS)}

TOPSIS (Technique for Order Preference by Similarity to Ideal Solution) was developed by Hwang and Yoon based on the concept that the chosen parameter should have the shortest distance from the best solution and the longest distance from the worst solution [20]. Normalized and weighted normalised values are shown in Table 3. Positive ideal, Negative ideal solutions, separation measures, closeness coefficient values, and rank are given in Table 4 .

\subsection{Step 1}

The normalized value $\left(\mathrm{r}_{\mathrm{ij}}\right)$ is obtained using the equation (1).

$$
\underset{\mathrm{ij}}{\mathrm{r}}=\frac{\mathrm{X}_{\mathrm{ij}}}{\sqrt{\sum_{\mathrm{i}=1}^{\mathrm{m}} \mathrm{X}_{\mathrm{ij}}^{2}}} \mathrm{i}=1,2,3 \ldots \ldots .32 ; j=1,2,3 .
$$

\subsection{Step 2}

By multiplying the normalized value with related weights the weighted normalized value $\left(v_{i j}\right)$ is calculated and is shown in equation (2),

$$
v_{i j}=w_{j} * r_{i j} i=1,2,3 \ldots 32 ; j=1,2,3 .
$$


3.3. Step 3

Then the positive ideal solution $\left(\mathrm{S}^{+}\right)$and negative ideal solution $\left(\mathrm{S}^{-}\right)$calculated using equation (3),

$S^{+}=\left\{\left(\operatorname{Max}\left(v_{i j}\right) \mid j \in J\right),\left(\operatorname{Min}\left(v_{i j}\right) \mid j \in J^{\prime}\right) \mid i=1,2 \ldots 32\right\}$

$S^{-}=\left\{\left(\operatorname{Min}\left(v_{i j}\right) \mid j \in J\right),\left(\operatorname{Max}\left(v_{i j}\right) \mid j \in J^{\prime}\right) \mid i=1,2 \ldots 32\right\}$

\subsection{Step 4}

The separation of each alternative from positive ideal solution $\left(\mathrm{S}^{+)}\right.$and negative ideal solution $\left(\mathrm{S}^{-}\right)$is found as per equation (4) and equation (5),

$$
\begin{gathered}
D_{i}^{+}=\sqrt{\sum_{i=1}^{32}\left(v_{i j-} s_{j}^{+}\right)^{2}} \mathrm{i}=1,2 \ldots 32, \\
D_{i}^{-}=\sqrt{\sum_{i=1}^{32}\left(v_{i j-} s_{j}^{-}\right)^{2}} \mathrm{j}=1,2,3 .
\end{gathered}
$$

\subsection{Step 5}

The closeness coefficient value of each alternative $\left(\mathrm{C}_{\mathrm{i}}\right)$ is calculated using equation (6),

$$
C_{i}=\frac{D_{i}^{-}}{D_{i}^{-}+D_{i}^{+}} .
$$

\begin{tabular}{|c|c|c|c|c|c|c|c|}
\hline $\begin{array}{l}\text { Exp. } \\
\text { No }\end{array}$ & $v$ & $f$ & $\begin{array}{c}d \\
(\mathrm{~mm})\end{array}$ & $\begin{array}{c}r \\
(\mathrm{~mm})\end{array}$ & $\begin{array}{c}\alpha \\
\left({ }^{\circ}\right)\end{array}$ & $\begin{array}{l}F_{m} \\
(\mathrm{~N})\end{array}$ & $\begin{array}{c}R a \\
(\mu \mathrm{m})\end{array}$ \\
\hline 1 & 400 & 0.04 & 0.5 & 0.6 & 35 & 404.7 & 0.52 \\
\hline 2 & 800 & 0.04 & 0.5 & 0.6 & 15 & 233.4 & 0.46 \\
\hline 3 & 400 & 0.08 & 0.5 & 0.6 & 15 & 322.1 & 0.45 \\
\hline 4 & 800 & 0.08 & 0.5 & 0.6 & 35 & 473.0 & 0.54 \\
\hline 5 & 400 & 0.04 & 0.7 & 0.6 & 15 & 317.4 & 0.55 \\
\hline 6 & 800 & 0.04 & 0.7 & 0.6 & 35 & 376.3 & 0.50 \\
\hline 7 & 400 & 0.08 & 0.7 & 0.6 & 35 & 583.0 & 0.53 \\
\hline 8 & 800 & 0.08 & 0.7 & 0.6 & 15 & 380.4 & 0.47 \\
\hline 9 & 400 & 0.04 & 0.5 & 1 & 15 & 273.5 & 0.48 \\
\hline 10 & 800 & 0.04 & 0.5 & 1 & 35 & 425.4 & 0.40 \\
\hline 11 & 400 & 0.08 & 0.5 & 1 & 35 & 561.1 & 0.50 \\
\hline 12 & 800 & 0.08 & 0.5 & 1 & 15 & 350.2 & 0.50 \\
\hline 13 & 400 & 0.04 & 0.7 & 1 & 35 & 443.7 & 0.50 \\
\hline 14 & 800 & 0.04 & 0.7 & 1 & 15 & 323.6 & 0.40 \\
\hline 15 & 400 & 0.08 & 0.7 & 1 & 15 & 411.7 & 0.60 \\
\hline 16 & 800 & 0.08 & 0.7 & 1 & 35 & 523.3 & 0.49 \\
\hline 17 & 200 & 0.06 & 0.6 & 0.8 & 25 & 430.8 & 0.55 \\
\hline 18 & 1000 & 0.06 & 0.6 & 0.8 & 25 & 355.4 & 0.45 \\
\hline 19 & 600 & 0.02 & 0.6 & 0.8 & 25 & 309.5 & 0.46 \\
\hline 20 & 600 & 0.1 & 0.6 & 0.8 & 25 & 534.4 & 0.53 \\
\hline 21 & 600 & 0.06 & 0.4 & 0.8 & 25 & 344.4 & 0.45 \\
\hline 22 & 600 & 0.06 & 0.8 & 0.8 & 25 & 449.2 & 0.48 \\
\hline 23 & 600 & 0.06 & 0.6 & 0.4 & 25 & 359.3 & 0.51 \\
\hline 24 & 600 & 0.06 & 0.6 & 1.2 & 25 & 446.2 & 0.48 \\
\hline 25 & 600 & 0.06 & 0.6 & 0.8 & 5 & 279.9 & 0.48 \\
\hline 26 & 600 & 0.06 & 0.6 & 0.8 & 45 & 601.2 & 0.50 \\
\hline 27 & 600 & 0.06 & 0.6 & 0.8 & 25 & 358.5 & 0.50 \\
\hline 28 & 600 & 0.06 & 0.6 & 0.8 & 25 & 370.7 & 0.51 \\
\hline 29 & 600 & 0.06 & 0.6 & 0.8 & 25 & 378.5 & 0.52 \\
\hline 30 & 600 & 0.06 & 0.6 & 0.8 & 25 & 403.9 & 0.51 \\
\hline 31 & 600 & 0.06 & 0.6 & 0.8 & 25 & 380.2 & 0.48 \\
\hline 32 & 600 & 0.06 & 0.6 & 0.8 & 25 & 370.6 & 0.52 \\
\hline
\end{tabular}

Tab. 2 Experimental matrix with responses
Tab. 3 Normalized and weighted normalised value

\begin{tabular}{ccccc}
\hline & \multicolumn{2}{c}{ Normalized value } & \multicolumn{2}{c}{ Weighted Normalized } \\
Exp. & \multicolumn{2}{c}{ value } \\
\cline { 2 - 5 } & $\begin{array}{c}\text { Machining } \\
\text { Force }\end{array}$ & $\begin{array}{c}\text { Surface } \\
\text { Roughness }\end{array}$ & $\begin{array}{c}\text { Machining } \\
\text { Force }\end{array}$ & $\begin{array}{c}\text { Surface } \\
\text { Roughness }\end{array}$ \\
\hline 1 & 0.17501 & 0.18532 & 0.08750 & 0.09266 \\
2 & 0.10095 & 0.16414 & 0.05047 & 0.08207 \\
3 & 0.13928 & 0.15991 & 0.06964 & 0.07995 \\
4 & 0.20454 & 0.19238 & 0.10227 & 0.09619 \\
5 & 0.13728 & 0.19486 & 0.06864 & 0.09743 \\
6 & 0.16275 & 0.17897 & 0.08137 & 0.08948 \\
7 & 0.25211 & 0.19027 & 0.12605 & 0.09513 \\
8 & 0.16449 & 0.16626 & 0.08224 & 0.08313 \\
9 & 0.11830 & 0.17120 & 0.05915 & 0.08560 \\
10 & 0.18397 & 0.14155 & 0.09198 & 0.07077 \\
11 & 0.24265 & 0.17897 & 0.12132 & 0.08948 \\
12 & 0.15146 & 0.17720 & 0.07573 & 0.08860 \\
13 & 0.19189 & 0.17932 & 0.09594 & 0.08966 \\
14 & 0.13993 & 0.14402 & 0.06996 & 0.07201 \\
15 & 0.17806 & 0.21321 & 0.08903 & 0.10660 \\
16 & 0.22631 & 0.17579 & 0.11315 & 0.08789 \\
17 & 0.18629 & 0.19733 & 0.09314 & 0.09866 \\
18 & 0.15369 & 0.16097 & 0.07684 & 0.08048 \\
19 & 0.13387 & 0.16520 & 0.06693 & 0.08260 \\
20 & 0.23111 & 0.18709 & 0.11555 & 0.09354 \\
21 & 0.14893 & 0.15885 & 0.07446 & 0.07942 \\
22 & 0.19424 & 0.16944 & 0.09712 & 0.08472 \\
23 & 0.15540 & 0.18144 & 0.07770 & 0.09072 \\
24 & 0.19295 & 0.17120 & 0.09647 & 0.08560 \\
25 & 0.12105 & 0.17085 & 0.06052 & 0.08542 \\
26 & 0.26000 & 0.17968 & 0.13000 & 0.08984 \\
27 & 0.15503 & 0.17897 & 0.07751 & 0.08948 \\
28 & 0.16031 & 0.18285 & 0.08015 & 0.09142 \\
29 & 0.16367 & 0.18356 & 0.08183 & 0.09178 \\
30 & 0.17468 & 0.18073 & 0.08734 & 0.09036 \\
31 & 0.16442 & 0.17226 & 0.08221 & 0.08613 \\
32 & 0.16027 & 0.18426 & 0.08013 & 0.09213 \\
\hline & & & & \\
& & & & \\
1 & & & \\
1 & & &
\end{tabular}

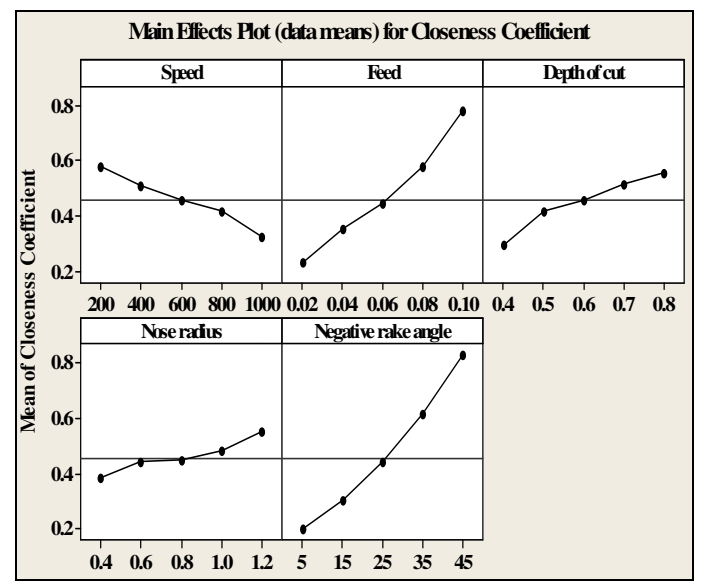

Fig. 3. Main effects plot 
Tab. 4 Separation measures, Closeness coefficient values and rank

\begin{tabular}{ccccccc}
\hline $\begin{array}{c}\text { Exp. } \\
\text { No }\end{array}$ & PIS & NIS & $\mathrm{D}_{\mathrm{i}}^{+}$ & $\mathrm{D}_{\mathrm{i}}^{-}$ & $\mathrm{C}_{\mathrm{i}}$ & Rank \\
\hline 1 & 0.08 & 0.09 & 0.04 & 0.04 & 0.490 & 12 \\
2 & 0.05 & 0.08 & 0.08 & 0.01 & 0.119 & 32 \\
3 & 0.07 & 0.08 & 0.06 & 0.02 & 0.244 & 27 \\
4 & 0.10 & 0.09 & 0.03 & 0.05 & 0.661 & 6 \\
5 & 0.06 & 0.09 & 0.06 & 0.03 & 0.342 & 24 \\
6 & 0.08 & 0.08 & 0.05 & 0.03 & 0.412 & 17 \\
7 & 0.12 & 0.09 & 0.01 & 0.07 & 0.867 & 1 \\
8 & 0.08 & 0.08 & 0.05 & 0.03 & 0.390 & 20 \\
9 & 0.05 & 0.08 & 0.07 & 0.01 & 0.189 & 31 \\
10 & 0.09 & 0.07 & 0.05 & 0.04 & 0.443 & 14 \\
11 & 0.12 & 0.08 & 0.01 & 0.07 & 0.792 & 3 \\
12 & 0.07 & 0.08 & 0.05 & 0.03 & 0.351 & 23 \\
13 & 0.09 & 0.09 & 0.03 & 0.04 & 0.564 & 8 \\
14 & 0.07 & 0.07 & 0.06 & 0.02 & 0.220 & 29 \\
15 & 0.08 & 0.10 & 0.04 & 0.05 & 0.562 & 9 \\
16 & 0.11 & 0.08 & 0.02 & 0.06 & 0.721 & 5 \\
17 & 0.09 & 0.09 & 0.03 & 0.05 & 0.575 & 7 \\
18 & 0.07 & 0.08 & 0.05 & 0.02 & 0.322 & 25 \\
19 & 0.06 & 0.08 & 0.06 & 0.02 & 0.231 & 28 \\
20 & 0.11 & 0.09 & 0.01 & 0.06 & 0.780 & 4 \\
21 & 0.07 & 0.07 & 0.06 & 0.02 & 0.292 & 26 \\
22 & 0.09 & 0.08 & 0.03 & 0.04 & 0.552 & 10 \\
23 & 0.07 & 0.09 & 0.05 & 0.03 & 0.382 & 21 \\
24 & 0.09 & 0.08 & 0.04 & 0.04 & 0.550 & 11 \\
25 & 0.06 & 0.08 & 0.07 & 0.01 & 0.197 & 30 \\
26 & 0.13 & 0.09 & 0.01 & 0.08 & 0.830 & 2 \\
27 & 0.07 & 0.08 & 0.05 & 0.03 & 0.373 & 22 \\
28 & 0.08 & 0.09 & 0.05 & 0.03 & 0.410 & 18 \\
29 & 0.08 & 0.09 & 0.05 & 0.03 & 0.428 & 15 \\
30 & 0.08 & 0.09 & 0.04 & 0.04 & 0.478 & 13 \\
31 & 0.08 & 0.08 & 0.05 & 0.03 & 0.404 & 19 \\
32 & 0.08 & 0.09 & 0.05 & 0.03 & 0.413 & 16 \\
\hline & & & & & & \\
\hline
\end{tabular}

Tab. 5 Mean response table for Closeness Coefficient

\begin{tabular}{cccccc}
\hline Level & \multicolumn{5}{c}{ Factor } \\
\hline & $v$ & $\mathrm{f}$ & $\mathrm{d}$ & $\mathrm{r}$ & $\alpha$ \\
\hline 1 & 0.5748 & 0.2309 & 0.292 & 0.3817 & 0.1965 \\
2 & 0.5063 & 0.3473 & 0.41109 & 0.4407 & 0.3021 \\
3 & 0.4513 & 0.4431 & 0.45509 & 0.4488 & 0.4420 \\
4 & 0.4146 & 0.5735 & 0.5098 & 0.4802 & 0.6188 \\
5 & 0.3217 & 0.77977 & 0.5521 & 0.54988 & 0.8299 \\
Max-Min & 0.2530 & 0.5488 & 0.2601 & 0.1681 & 0.6334 \\
Rank & 4 & 2 & 3 & 5 & 1 \\
\hline
\end{tabular}

\section{RESULTS AND DISCUSSION}

The higher the value of closeness coefficient indicates better performance. From Table 4 , it is evident that the experiment number 7 having the highest value of closeness coefficient was the better performer amongst the 32 number of experiments.
The order of the experimental run obtained by TOPSIS was given by $7>26>11>20>16>4>17>$ $13>15>22>24>1>30>10>29>32>6>28>31>8>23>27>$ $12>5>18>21>3>19>14>25>9>2$.

Optimum closeness coefficients are observed (Shown in Fig.3.) at $v=200 \mathrm{rpm}, f=0.1 \mathrm{~mm} / \mathrm{rev}, d=$ $0.8 \mathrm{~mm}, r=1.2 \mathrm{~mm}$ and $\alpha=45^{\circ}$ and similar observations are made from mean response table for closeness coefficient shown in Table 5 .

Tab. 6 ANOVA for Closeness Coefficient

\begin{tabular}{|c|c|c|c|c|c|c|c|}
\hline Source & DF & Seq SS & $\begin{array}{l}\text { Adj } \\
\text { SS } \\
\end{array}$ & $\begin{array}{l}\text { Adj } \\
\text { MS }\end{array}$ & $\mathrm{F}$ & $\mathrm{P}$ & $\begin{array}{l}\% \\
\mathrm{C}\end{array}$ \\
\hline$v$ & 1 & 0.064 & 0.003 & 0.003 & 1.8 & 0.19 & 5.36 \\
\hline $\mathrm{f}$ & 1 & 0.352 & 0.007 & 0.007 & 4.3 & 0.06 & 29.48 \\
\hline d & 1 & 0.072 & 0.009 & 0.009 & 5.8 & 0.03 & 5.99 \\
\hline $\mathrm{r}$ & 1 & 0.018 & 0.000 & 0.000 & 0.0 & 0.79 & 1.48 \\
\hline$\alpha$ & 1 & 0.602 & 0.009 & 0.009 & 5.5 & 0.03 & 50.36 \\
\hline$v^{*} v$ & 1 & 0.000 & 0.000 & 0.000 & 0.2 & 0.65 & 0.00 \\
\hline$v^{*} \mathrm{f}$ & 1 & 0.000 & 0.000 & 0.000 & 0.09 & 0.76 & 0.01 \\
\hline$v^{*} \mathrm{~d}$ & 1 & 0.013 & 0.013 & 0.013 & 8.2 & 0.015 & 1.07 \\
\hline$v^{*} \mathrm{r}$ & 1 & 0.000 & 0.000 & 0.000 & 0.01 & 0.93 & 0.00 \\
\hline$v^{*} \alpha$ & 1 & 0.003 & 0.003 & 0.003 & 1.9 & 0.18 & 0.26 \\
\hline$f * f$ & 1 & 0.008 & 0.009 & 0.009 & 5.8 & 0.03 & 0.63 \\
\hline $\mathrm{f}^{*} \mathrm{~d}$ & 1 & 0.002 & 0.002 & 0.002 & 1.5 & 0.23 & 0.20 \\
\hline$f^{*} \mathrm{r}$ & 1 & 0.003 & 0.003 & 0.003 & 1.8 & 0.20 & 0.24 \\
\hline $\mathrm{f}^{*} \alpha$ & 1 & 0.064 & 0.003 & 0.003 & 8.3 & 0.01 & 1.08 \\
\hline$d^{*} d$ & 1 & 0.352 & 0.007 & 0.007 & 0.2 & 0.66 & 0.07 \\
\hline $\mathrm{d}^{*} \mathrm{r}$ & 1 & 0.072 & 0.009 & 0.009 & 1.7 & 0.21 & 0.22 \\
\hline$d^{*} \alpha$ & 1 & 0.018 & 0.000 & 0.000 & 7.5 & 0.01 & 0.99 \\
\hline r*r & 1 & 0.602 & 0.009 & 0.009 & 1.1 & 0.31 & 0.09 \\
\hline $\mathrm{r}^{*} \alpha$ & 1 & 0.000 & 0.000 & 0.000 & 0.7 & 0.40 & 0.10 \\
\hline$\alpha^{*} \alpha$ & 1 & 0.000 & 0.000 & 0.000 & 7.2 & 0.02 & 0.94 \\
\hline Error & 11 & 0.013 & 0.013 & 0.013 & & & 1.43 \\
\hline Total & 31 & 1.194 & & & & & \\
\hline
\end{tabular}

In the response table (Table 5) it has shown that a negative rake angle has been assigned a rank 1 which means it is the most significant parameter in controlling the response followed by feed, depth of cut, cutting speed and nose radius.

From the ANOVA table 6, it is clear that the negative rake angle $(50.36 \%)$ has significant influence followed by feed (29.47\%), Depth of cut (5.98\%), speed $(5.36 \%)$ and nose radius $(1.48 \%)$ has least influence.

The Closeness coefficient for the obtained optimum combination of parameters was 1.463687 estimated from equation 7 and was $68.73 \%$ higher than the maximum Closeness coefficient corresponding to rank 1 in Table 4 . Hence the values obtained are optimum.

$$
\gamma=\gamma_{\mathrm{m}}+\sum_{\mathrm{i}=1}^{\mathrm{q}}\left(\overline{\gamma_{\mathrm{j}}}-\gamma_{\mathrm{m}}\right)
$$




\section{CONCLUSIONS}

Experiments were conducted as per CCD of RSM and optimized cutting parameters in AISI 52100 steel hard turning using TOPSIS.

1. The negative rake angle is the most significant parameter in controlling the response followed by feed, depth of cut, cutting speed and nose radius.

2. From the ANOVA negative rake angle (50.36\%) has significant influence followed by feed $(29.47 \%)$, Depth of cut $(5.98 \%)$, Speed $(5.36 \%)$ and Nose radius $(1.48 \%)$ has least influence.

3. It is clear from the results of TOPSIS experiment number 7 has the highest closeness coefficient value. Optimal parametric combinations are at speed $200 \mathrm{rpm}$, feed $0.1 \mathrm{~mm} / \mathrm{rev}$, depth of cut 0.8 $\mathrm{mm}$, nose radius $1.2 \mathrm{~mm}$ and negative rake angle $45^{\circ}$.

4. From the values of closeness coefficient, the machining parameters best combination can be arranged in the order $7>26>11>20>16>4>17>13>$ $15>22>24>1>30>10>29>32>6>28>31>8>23>27>$ $12>5>18>21>3>19>14>25>9>2$.

5. An improvement of $68.73 \%$ of the predicted weighted closeness coefficient establishes the optimality of obtained results.

\section{Acknowledgements}

Authors would like to thank Karunya Institute of Technology and sciences, Coimbatore for providing facilities.

\section{Nomenclature}

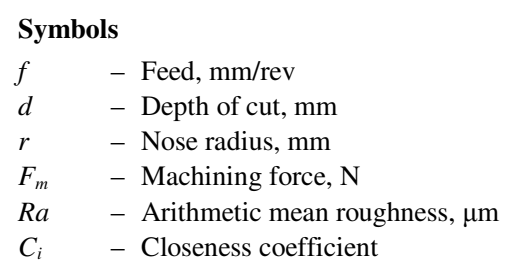

\section{Greek letters}

$$
\begin{array}{ll}
v & - \text { cutting speed, rpm } \\
\alpha & - \text { Negative rake angle, }\left(^{\circ}\right)
\end{array}
$$

\begin{tabular}{|c|c|c|}
\hline \multicolumn{2}{|c|}{ Acronyms } & \multirow[b]{2}{*}{ Central composite design } \\
\hline $\mathrm{CCD}$ & - & \\
\hline PCBN & - & Polycrystalline Cubic Boron Nitride \\
\hline AISI & - & American Iron and Steel Institute \\
\hline RSM & - & Response surface Method \\
\hline TOPSIS & - & $\begin{array}{l}\text { technique for order of preference by similarity } \\
\text { to ideal solution }\end{array}$ \\
\hline ANOVA & - & Analysis of Variance \\
\hline PIS & - & Positive ideal Solution \\
\hline NIS & - & Negative ideal Solution \\
\hline GRA & - & Grey relational approach \\
\hline PCA & - & Principle component analysis \\
\hline MCDM & - & Multi criteria decision making \\
\hline ANN & - & Artificial neural network \\
\hline DF & - & Degrees of freedom \\
\hline
\end{tabular}

\section{References}

1. König W., Hochschule T., Komanduri R., Schenectady D., Tönshoff H.K. (1984). Machining of hard materials. Ann CIRP, Vol. 33, No. 2, pp. 417-427.

2. Tönshoff H.K., Arendt C., Amor R.B. (2000). Cutting of hardened steel. Ann CIRP, Vol. 49, No. 2, pp. 547-566.

3. Mark Velasquez., Patrick T. Hester. (2013). An Analysis of Multi-Criteria Decision Making Methods. International Journal of Operations Research, Vol. 10, No. 2, pp. 56-66.

4. Balasubramaniyan Singaravel., Thangiah Selvaraj. (2015). Optimization of machining parameters in turning operation using combined TOPSIS and AHP method. Tehnički vjesnik, Vol. 22, No. 6, pp. 1475-1480.

5. Himadri Majumder., Abhijit Saha. (2018). Application of MCDM based hybrid optimization tool during turning of ASTM A588. Decision Science Letters, Vol. 7, pp. 143-156.

6. Tian Syung Lan. (2009). Taguchi optimization of Multi objective CNC Machining using TOPSIS. Information technology journal, Vol. 8, No. 6, pp. 917-922.

7. Palanisamy D., Senthil P. (2016). Optimization on Turning Parameters of 15-5PH Stainless Steel Using Taguchi Based Grey Approach and TOPSIS. Archive of Mechanical Engineering, LXIII (3), pp. 397- 412.

8. Maheswararao Ch., Venkata subbaiah K. (2016). Application of WSM, WPM and TOPSIS methods for the optimization of multiple responses. International journal of hybrid information technology, Vol. 9, No. 10, pp. 59-72.

9. Sagar Bhise P., Pantanwane P.D., Rajiv B. (2014). Optimisation of Hard Turning of M42 Tool Steel Using PCR-TOPSIS Method. $5^{\text {th }}$ International \& $26^{\text {th }}$ All India Manufacturing Technology, Design and Research Conference (AIMTDR 2014) IIT Guwahati, Assam, India, $12^{\text {th }}-14^{\text {th }}$ December.

10. Maity K., Khan A. (2017). Application of MCDM-based TOPSIS method for the selection of Optimal Process Parameter in Turning of Pure Titanium. Benchmarking: An International Journal, Vol. 24, No. 7, pp. 2009-2021.

11. Singaravel B., Selvaraj T., Vinodh S. (2016). multiobjective optimization of turning parameters using the combined MOORA and entropy method. Transactions of the Canadian Society for Mechanical Engineering, Vol. 40, No.1, pp. 101-111.

12. Singaravel B., Prabhu Shankar D., Lakshmi Prasanna. (2018). Application of MCDM Method for the Selection of Optimum Process Parameters in Turning Process. Materials Today: Proceedings, Vol. 5, No. 5, pp. 13464-13471.

13. Umamaheswarrao P., Ranga Raju D., NS Suman K.., Ravi Sankar B. (2018). Multi objective optimization of Process parameters for hard turning of AISI 52100 steel using Hybrid GRA-PCA. Procedia Computer Science, Vol. 133, pp. 703-710.

14. Umamaheswarrao P., Ranga Raju D., NS Suman K., Ravi Sankar B. (2019). Parametric optimization of surface roughness and workpiece surface temperature during hard turning of AISI 52100 steel using Hybrid GRA-PCA. In: $2^{\text {nd }}$ International Conference on Computational Methods in Manufacturing, (ICCMM 2019) Indian Institute of Technology Guwahati, India, 89 March.

15. Umamaheswarrao P., Ranga Raju D., NS Suman K., Ravi Sankar B. (2019). Achieving optimal process parameters during Hard Turning of AISI 52100 Bearing Steel using Hybrid GRA-PCA. Key Engineering Materials, Vol. 818, pp. 87-91.

16. Serra R., Chibane H., and Duchosal A. (2018). Multiobjective optimization of cutting parameters for turning AISI 52100 hardened steel. International Journal of 
Advanced Manufacturing Technology, Vol. 99, pp. 20252034.

17. UmaMaheshwera Reddy P., Harish D., Suresh Kumar Reddy N. (2018). Application of Regression and Artificial Neural Network Analysis in Modelling of Surface Roughness in Hard Turning of AISI 52100 Steel. Materials Today: Proceedings, Vol. 5, No. 1, pp. 47664777.

18. Swapna D., Srinivasa Rao Ch., Kumar S., Radhika S. (2019). AHP and TOPSIS Based Selection of Aluminium Alloy for Automobile Panels. Journal of Mechanical and Energy Engineering, Vol. 3(43), No. 1, pp. 43-50.

19. Umamaheswarrao P., Ranga Raj D., NS Suman K., Ravi Sankar B. (2019). TOPSIS Based Optimization of Process Parameters While Hard Turning of AISI 52100 Steel. Acta Mechanica Malaysia, Vol. 2, Issue. 2, pp. 28-31.

20. Yoon K Paul, and Ching-Lai Hwang. (1995). Multiple attribute decision making: an introduction. Sage publications.

\section{Biographical notes}

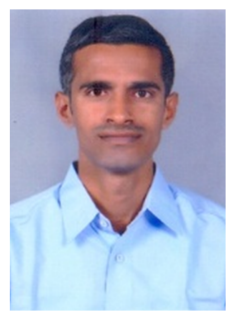

P. Umamaheswarrao obtained his M.Tech from Vellore Institute of Technology, Vellore. Currently he is working as an Assistant professor in the Department of Mechanical Engineering, Bapatla Engineering College, Bapatla, Andhra Pradesh. He has presented papers in various international conferences.

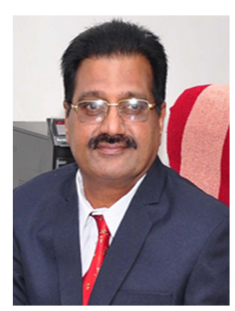

Dr. D. Ranga Raju Received his M.Tech and Ph.D from IIT Kharagpur. At present he is working as a professor in the Department of Mechanical Engineering, Srinivasa Institute of Engineering and Technology, Amalapuram, Andhra Pradesh. He has vast experience in teaching and administration.

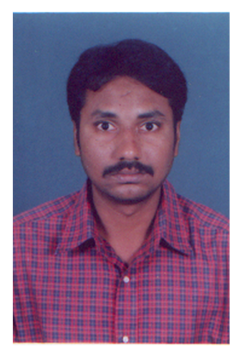

Dr. Koka Naga Sai Suman is working as an Associate Professor in the Department of Mechanical Engineering, Andhra University College of Engineering, Visakhapatnam. He received best thesis award for his $\mathrm{Ph} . \mathrm{D}$ work in the year 2010 by Andhra University. His areas of interests include Advanced Composites, Nano technology, Manufacturing.

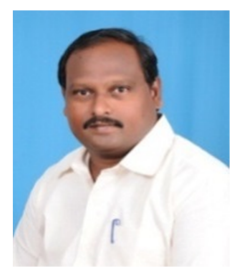

Dr. B. Ravi Sankar working as a professor in the Department of Mechanical Engineering, Bapatla Engineering College, Bapatla, A.P. He obtained his Ph.D from Andhra University, Visakhapatnam. His scientific interests focus on Nanofluids, Alternative fuels, Machinabilty studies of hard materials, Composite materials, Friction stir welding. He has presented papers in various international conferences. 